ACTA UNIVERSITATIS LODZIENSIS

Folia Litteraria Romanica 14, 2019

http://dx.doi.org/10.18778/1505-9065.14.11

Matgorzata Izert

Université de Varsovie

(iD https://orcid.org/0000-0002-0212-3966

m.izert@uw.edu.pl

\title{
Une larme de cognac et un soupçon de lait - à propos de quelques quantifieurs nominaux marquant une petite quantité
}

\begin{abstract}
RÉSUMÉ
Dans cet article nous analysons des collocations qui constituent la suite $D E T N_{1}$ de $N_{2}$ et servent à exprimer habituellement une (très) petite quantité ou une quantité minime de liquide. Les paraphrases de ce type de collocations données par les dictionnaires de langue comme 'petite quantité de N', 'un peu de N' ne renvoient qu'à une partie du sens apporté par ces quantifieurs nominaux dont le sens exact est beaucoup plus complexe et nuancé. Par exemple, une pointe de whisky indique non seulement une petite quantité d'alcool mais désigne aussi une intensité faible de son goût. De même, la combinatoire de ces collocatifs attestée par les dictionnaires de langue n'est pas aussi large que celle confirmée par l'usage écrit récent dans les textes provenant des données numérisées. Ces données permettent de distinguer quelques types de $N_{2}$ d'après la nature des objets de référence qu'ils désignent ( $c f$. toutes sortes de boissons (y compris les alcools) ou toutes sortes de produits liquides mais aussi les produits massifs alimentaires ou non alimentaires et les idées abstraites - sentiments, émotions, etc. et même, ce qui peut paraître surprenant, les styles musicaux).
\end{abstract}

MOTS-CLÉS - collocation, quantification, quantifieur nominal, quantité, intensité

\section{Une larme de cognac and un soupçon de lait: About Some of the Nominal Quantifiers That Indicate Small Quantity}

\section{SUMMARY}

In this paper, I wish to present the analysis of collocations DET $N_{1}$ ' $d e^{\prime} \mathrm{N}_{2}$ in French, which is usually used to denote small or minimal amounts of a liquid. Dictionary paraphrases of the collocation referring to a 'small amount of N' (fr. 'petite quantité de $N$ ') or 'some N' (fr. 'un peu de $N$ ') do not always describe the meaning and value of these expressions accurately. For example, 'une pointe de whisky' indicates not only a very small amount of alcohol, but also a delicate touch of taste and, thus, a low degree of its intensity. Also the connectivity of DET $\mathrm{N}_{1}$ ' $d e$ ' with $\mathrm{N}_{2}$ described in dictionaries differs from the one found in the actual contemporary text use. DET $\mathrm{N}_{1}$ ' $d e$ ' connects not only with nouns that mean different types of drinks (including alcohol/alcoholic) or liquids, but also with nouns denoting non-countable solids or abstract concepts referring most often to feelings and emotions as well as, surprisingly, music.

KEYWORDS - collocation, substantive quantifier, quantity, intensity 


\section{Introduction}

L'objectif de cette étude sera d'analyser les collocations qui constituent la suite $D E T N_{1}$ de $N_{2}$ et servent à exprimer principalement une (très) petite quantité ou une quantité minime de liquide. Dans ce type de collocations le premier nom $-N_{1}$ est associé aux noms sans déterminant $-N_{2}$ (étant bases de collocation) et fonctionne comme un marqueur de quantité plus ou moins déterminée ( $c f$. une goutte de jus d'orange, 3 gouttes de probiotique, une gorgée d'eau, un doigt de vin) ou s'interprète comme un marqueur de quantité indéterminée ( $c f$. une larme de porto, un soupçon de crème, un nuage de lait).

Nous essayerons aussi de répondre à quelques questions fondamentales : Estce que les paraphrases 'petite quantité de N' ou 'un peu de N' sont suffisantes pour exprimer le sens apporté par ce type de collocations ? Ne perdent-elles pas de leurs traits connotatifs ? Est-ce qu'il y a des traits communs à certains types de collocatifs quantifieurs? Est-ce qu'on peut substituer chacun de ces collocatifs par un autre collocatif, par exemple un nuage de par une larme de pour le nom de base lait ? Est-ce que ce sont uniquement les noms de liquides qui privilégient ce type de quantifieurs? Et enfin, pourquoi les mêmes pensées nous paraissentelles beaucoup plus vives et en même temps précises quand elles sont exprimées par une figure plutôt que par des locutions toutes simples comme 'un peu de' ou 'petite quantité de'?

\section{L'objet d'étude et les sources d'investigation}

L'inventaire des quantifieurs nominaux que nous avons sélectionnés pour cette étude comporte huit groupes nominaux tels un doigt de (ex. un doigt de rhum), une gorgée de (ex. une gorgée de café), une goutte de (ex. une goutte de jus de citron), une larme de (ex. une larme de cognac), un nuage de (ex. un nuage de lait), une pointe de (ex. une pointe de vinaigre), un soupçon de (ex. un soupçon de crème), une trace de (ex. une trace d'alcool).

Comme source d'investigation nous avons choisi, pour le corpus préliminaire le Trésor de la Langue Française informatisé (TLFi) et pour le corpus d'analyse des emplois un gros volume textuel sur un support électronique qui est le Web français ${ }^{1}$ permettant d'effectuer des études centrées sur de nouvelles associations de lexèmes (leurs formes et leurs sens) non enregistrées par les dictionnaires mais comportant un nombre important d'occurrences, sur leurs régularités, leur fréquence et leur contexte d'emploi - impossible à observer dans les dictionnaires de langue.

\footnotetext{
${ }^{1}$ Consulté entre le 6.05.2018 et le 31.05.2018.
} 


\section{Quelques généralités et précisions terminologiques}

Préalablement, nous exposerons brièvement les choix terminologiques et les principales caractéristiques de ce type d'associations d'éléments lexicaux.

\subsection{Définition : quantifieurs nominaux}

La suite : $D E T N_{1}$ de est couramment appelée déterminant nominal (Dessaux, 1976; Leroy, 2005), déterminant nominal quantifieur (Buvet, 1993), substantif quantificateur (Benninger, 1999) ou quantifieur nominal (Maingueneau, 1994 ; Asnès, 2008 : 82) ou encore quantifieur d'origine nominale (Gaatone, 2013 : 43). Nous appelons ce type de constructions quantifieurs nominaux (Izert, 2018) et nous les définissons comme marqueurs formels d'amplification des objets de référence désignés par les noms auxquels ces marqueurs sont adjoints.

\subsubsection{Marqueurs de quantité plus ou moins déterminée}

Trois quantifieurs nominaux qui font l'objet de cette étude servent à exprimer une quantité déterminée, cf. une goutte d'arôme naturel de bergamote ou une quantité plus ou moins déterminée, $c f$. une gorgée de thé tiède, un doigt de vin mais non précise quant à la quantité exprimée en unités de mesure / de volume (quantité en millilitres).

\subsubsection{Marqueurs de quantité indéterminée}

Cinq quantifieurs nominaux marquent une quantité indéterminée, c'est-à-dire la quantité qui ne peut pas être mesurée et qui n'est donc pas exprimée par un nombre précis, $c f$. une larme de scotch, un nuage de lait, une pointe de vinaigre, un soupçon de crème, une trace d'alcool.

\subsubsection{Marqueurs de petite quantité / de quantité faible}

Tous ces quantifieurs nominaux, aussi bien ceux qui marquent d'habitude une quantité plus ou moins déterminée que ceux qui marquent une quantité indéterminée, désignent une quantité qui est au-dessous de la norme et un peu au-dessus de zéro ou tend vers zéro, ils marquent une très petite quantité (quantité très faible) ou une quantité minime.

\subsection{Collocatifs figuratifs ou métaphoriques}

Les expressions construites avec ces quantifieurs constituent les collocations, c'est-à-dire « les cooccurrences lexicales privilégiées de deux éléments linguistiques entretenant une relation syntaxique $»^{2}$ (Mel'cuk, 1984 ; Haussman, 1989). Certains

\footnotetext{
${ }^{2}$ Définition étroite largement privilégiée parmi les lexicologues et les lexicographes.
} 
parmi les collocatifs quantifieurs sont figuratifs ( $c f$. une larme de cognac, un doigt de vin, un soupçon de lait, etc.) parce qu'ils « ne fonctionnent jamais comme quantifieurs en dehors de leur emploi figuré »" (Blanco, 2002 : 69) ou ils peuvent acquérir une valeur métaphorique, quand ils s'écartent de l'usage ordinaire, habituel de la langue ( $c f$. une goutte de lait, une gorgée de thé) pour donner une signification et une expressivité particulières au propos ( $c f$. une goutte d'amour, une gorgée de liberté).

\section{Analyse de la combinatoire des collocatifs quantifieurs}

On peut distinguer deux sous-classes de collocatifs quantifieurs dans lesquelles les DET $N_{1}$ de sont regroupés selon leurs traits sémantiques et leur combinatoire avec les noms qui privilégient ce type de quantifieurs : collocatifs « liquides » et collocatifs « alimentaires » ou « non alimentaires ».

\subsection{Collocatifs « liquides »}

Trois quantifieurs collocatifs : une gorgée de, une goutte de, une larme de ayant un trait spécifique [+liquide] marquent avant tout une (très) petite quantité ou encore une quantité minime de liquide, c'est-à-dire une quantité faible de boissons, y compris de boissons alcoolisées, et de produits alimentaires liquides, par exemple :

(1) Et je me penchai pour avaler une gorgée de soupe sous son regard attentif.

(2) Sur chaque palourde, disposer : un peu de cerfeuil, une goutte de jus de citron vert, une goutte d'huile de Cumbavas. Servir de suite !

(3) Au cas où le résultat serait trop acide pour le bébé ( ), vous pouvez ajouter une larme de sirop d'agave.

Un doigt de, bien qu'il n'ait pas de trait spécifique [+liquide] s'emploie avant tout avec des noms désignant les boissons, par exemple :

(4) Une façon délicieuse de finir un bon repas. Ajoutez tout simplement un doigt de whisky irlandais et un nuage de Bailey's dans un bon café, et voilà, le tour est joué !

(5) A servir très frais, en apéritif, avec un doigt de sirop de pamplemousse ou nature pour accompagner les plats de votre été : salade, sushis, spécialités...

Les traits lexicaux communs pour tous les noms $N_{2}$ qui acceptent les collocatifs " liquides » sont donc [+concret] [+massif] [+liquide]. Les définitions de ces collocatifs ainsi que les exemples d'emploi qu'on trouve dans les dictionnaires de langue ${ }^{3}$ et dans les textes provenant du Web français le confirment.

${ }^{3}$ une goutte de : A. - Très petite quantité de liquide de forme arrondie (TLFi). une gorgée de : A. - [Constr. avec un compl. prép. de désignant un liquide] Quantité de liquide avalée en un seul 


\subsection{Collocatifs « alimentaires » ou « non alimentaires»}

D'après les définitions dictionnairiques quatre quantifieurs collocatifs : un nuage de, une pointe de, un soupçon de et une trace de $e^{4}$ marquent, entre autres, une petite quantité ou une quantité minime de liquide ( $c f$. une pointe de vinaigre, un soupçon de lait, un nuage de crème, une trace d'alcool) mais ils peuvent également indiquer une très petite quantité d'autres produits massifs alimentaires ou non alimentaires ( $c f$. une pointe de poivre, un soupçon de rouge à lèvres, un nuage de poudre, une trace d'or), par exemple :

(6) Si vous supportez mal la caféine et que vous voulez réussir à dormir paisiblement, c'est ce qu'il vous faudra commander. Il s'agit en fait d'une tasse de lait agrémenté d'un nuage de café.

(7) Une nouvelle recette pour la gamme Velours de Crème à base d'Oignons, un des légumes incontournable de notre Sud. Mais pour ne pas le laisser seul, et pour surprendre vos papilles, le voici en compagnie d'une pointe de piment fort, et pour adoucir les angles un nuage de miel.

(8) Le destinataire goûte les chocolats, qui contiennent un soupçon de lait, de caramel, de noix, de fruits, de fleurs, de vanille et de cacao.

(9) Ce pinceau court, aux poils ultra-doux, est parfait pour balayer un soupçon de poudre de façon homogène sur le visage.

(10) Pour dynamiser la digestion ou soulager des nausées, faire de même ou mettre une trace d'huile pure sur la langue.

Pourtant la combinatoire de ces collocatifs attestée par les dictionnaires de langue n'est pas aussi large que celle confirmée par l'usage écrit récent dans les textes provenant des données numérisées. Ces données ont permis de distinguer quelques types de $N_{2}$ d'après la nature des objets de référence qu'ils désignent. Ce sont :

- des noms de boissons et de produits alimentaires liquides, par exemple :

un doigt de sirop, une gorgée de bouillon, une goutte de jus de citron, une larme de sirop, un nuage de lait, une pointe de vinaigre, un soupçon de miel, une trace d'huile de coco, etc.

mouvement de déglutition (TLFi).

une larme de: III. P. anal. A. - Petite quantité, goutte de liquide (TLFi).

un doigt de : II. - P. méton. Unité de mesure grossièrement évaluée à l'épaisseur d'un doigt. [...]

P. ext. En petite quantité (TLFi).

${ }^{4}$ un nuage de : Petite quantité se répandant en évoquant un peu la légèreté d'un nuage. Synon. soupçon (de). Nuage de poudre, de crème, de lait (TLFi).

une pointe de : D. - 1. a) Petite quantité d'un condiment à saveur piquante (TLFi). un soupçon de : Quantité minime de quelque chose. [...] (pop. et fam.). Soupçon de crème, de poivre, de thym. (TLFi).

une trace de: Très faible taux d'une substance que l'on découvre, à l'analyse, dans une autre substance (TLFi). 
- des noms de boissons alcoolisées, par exemple :

un doigt de vin, une goutte d'armagnac, une larme de porto, un nuage de gin, une gorgée d'absinthe, une pointe de kirsch, un soupçon de whisky, une trace de rhum, etc.

- des noms de produits alimentaires massifs non liquides ${ }^{5}$, par exemple : une soupçon de sel, une pointe de persil, un nuage de sucre, une trace de chocolat, etc.

- des noms de produits non alimentaires plus ou moins liquides, par exemple : une larme de cire chaude, une goutte de poix, un soupçon de sang, une trace de boue, un nuage de bave, une pointe de peinture, une gorgée d'huile de paraffine, etc.

- des noms de produits non alimentaires plus ou moins solides, massifs, par exemple :

un nuage de poudre, un soupçon de rouge à lèvre, une trace d'or, un doigt de potasse, une pointe d'air, une gorgée de fumée, une larme de feu, etc.

- des noms de termes abstraits, tels les sentiments, les émotions, les états d'âme, etc. ${ }^{6}$, par exemple :

un doigt de bon sens, une gorgée d'optimisme, une goutte de magie, un nuage de tendresse, une pointe de jalousie, un soupçon de joie maligne, une trace d'espoir, etc.

- des noms de style musical, par exemple :

une larme de pop rock, un nuage de punk, un soupçon de métal, une pointe / une goutte/un doigt de jazz, une pincée de pop, une trace de rock gothique, une gorgée de jazz et de soul, une larme de pop, etc.

- des noms propres (noms de vins ou de domaines), par exemple :

(11) Château Minuty, une gorgée de Saint-Tropez. Ce n'est pas du vin, c'est du rosé !

(12) Une gorgée de Géorgie ? En 2011, Stéphane Bannwarth devenait le premier vigneron français à vinifier dans des jarres en terre cuite de Géorgie.

Les données provenant des pages Web ont fait émerger de nouvelles bases de collocation qui enrichissent le stock dictionnairique. Les mêmes données ont permis de vérifier la combinatoire de ces collocatifs. Cinq d'entre eux peuvent être

${ }^{5}$ À l'exception des collocatifs dits liquides (une goutte de, une gorgée de, une larme de).

${ }^{6}$ Le collocatif une larme de / des larmes de suivi de nom marquant un sentiment ou une émotion comme bonheur / joie / regret / tristesse n'a pas de valeur intensive. 
substitués l'un par l'autre pour des bases de collocation $N_{2}$ aussi bien [ + concret] [+massif] [+liquide] que [+concret] [+massif] [-liquide] ou encore [+abstrait]. Les collocatifs une goutte de, une gorgée de, une larme de ne sont jamais acceptés par les $N_{2}[+$ massif] [-liquide] à cause des contraintes sémantiques de sélection : *une larme de poivre, *une gorgée de sucre, *une goutte de sel, etc.

\section{La valeur sémantique de $D E T N_{1}$ de marquant une petite quantité}

Six quantifieurs sont issus du transfert figuratif, $c f$. une larme vs une larme de cognac, un nuage vs un nuage de lait, un doigt vs un doigt de sirop, un soupçon vs un soupçon de liqueur, etc., deux quantifieurs d'un transfert métaphorique, $c f$. une goutte d'eau vs une goutte d'amour, une gorgée de soupe vs une gorgée de liberté. Le figement sémantique des collocations construites avec ces quantifieurs n'est que partiel : un seul élément, $D E T N_{l} d e$ (collocatif), acquiert un sens figuré / métaphorique, l'autre, le $N_{2}$ (base de collocation), nom [+concret] [+massif] à quantifier ou nom [+abstrait] à intensifier, s'emploie au sens propre.

Tous les collocatifs quantifieurs peuvent être approximativement paraphrasés par 'un peu de N' ou 'une (très) petite quantité de N' mais ces paraphrases ne sont pas toujours suffisantes pour exprimer le sens apporté par les collocations construites avec ces quantifieurs.

Premièrement, la paraphrase 'une petite quantité de N' qu' on trouve d'habitude dans les dictionnaires de langue n'est pas trop précise. Un peu de lait ou une petite quantité de lait peuvent signifier, selon les contextes, 'un verre de lait', 'une cuillerée de lait', 'une gorgée de lait' ou 'une goutte de lait', etc. Dans le cadre de la sémantique évaluative, un peu de ou une petite quantité de posent, d'une part, l'existence d'une certaine quantité indéterminée et présente celle-ci comme faible, elle est située au-dessous de la norme mais au-dessus de la quantité nulle. De même, tous les collocatifs qui font l'objet de notre étude servent à marquer une quantité qui dépasse faiblement le niveau zéro. Il s'agit d'une petite quantité ou d'une toute petite quantité : 'un tout petit peu'(cf. une gorgée de, un doigt de, par ex. Donnez-moi du vin mais je n'en veux qu'un doigt) ou d'une quantité minime (cf. une goutte de, une larme de, un nuage de, une pointe de, un soupçon de, une trace de). Dans certains contextes d'emploi la quantité exprimée par ce type de collocatifs peut être jugée insuffisante, par ex. Elle a bu une gorgée de bouillon et a mangé une bouchée de pain, c'est-à-dire qu'elle a bu et a mangé très peu ou trop peu ou, au contraire, suffisante : Versez une larme de vodka dans votre café et vous vous réchaufferez. Une goutte d'amour dans un océan de haine, et le monde peut être sauvé.

Deuxièmement, 'un peu de' ou 'une petite quantité de' ne renvoient qu'à une partie du sens apporté par certains collocatifs quantifieurs. Leur sens exact est plus nuancé et ne se réduit pas seulement à l'idée de quantité. Leur interprétation 
sémantique dépend à la fois de la nature et de la classe d'appartenance sémantiques de $N_{2}$ et des nuances de sens spécifiques véhiculées par chaque collocatif.

Dans un soupçon de lait / de thé, un soupçon de marque 'une quantité minime de lait / de thé' presque non perceptible au goût, dans un soupçon d'accent étranger - une apparence légère, faiblement perceptible à l'oreille et dans un soupçon de moustaches une apparence faiblement perceptible à l'œil. Les collocations avec une trace de (cf. une trace d'alcool dans une boisson, une trace de poivre dans un plat, une trace d'accent étranger, etc.) prennent les mêmes nuances de sens. Elles expriment une quantité à peine décelable dans une autre substance.

Un nuage de lait désigne une très petite quantité de lait qui se répand en évoquant un peu la légèreté d'un nuage ( $c f$. un café nappé d'un nuage de crème fraîche, un thé avec un nuage de miel, etc.).

Une pointe de whisky indique à peu près la même quantité qu'une goutte de whisky - une toute petite quantité ou une quantité minime mais ce collocatif peut aussi désigner une intensité faible de goût d'un liquide ou d'un produit alimentaire (cf. confiture de framboises avec une pointe de whisky, une gourde avec une pointe de menthe, etc.).

Une gorgée de soupe renvoie à une petite quantité de liquide avalée en un seul mouvement de déglutition.

Un doigt de vin ou de sirop semble marquer non seulement une très petite quantité de boisson mais aussi une épaisseur faible (du doigt) indiquant le niveau dans le verre.

Troisièmement, toutes les collocations où le collocatif est suivi de $N_{2}$ [+abstrait $]$ ne peuvent être interprétées que de manière non quantitative. Le collocatif indique une intensité faible d'une propriété par rapport à son état neutre, une norme ( $c f$. un soupçon de joie maligne, une pointe de jalousie, une goutte de bonheur, un nuage de gaieté, etc.) ce qui n'est pas mentionné par les dictionnaires de langue.

Il nous reste encore à répondre à la dernière question posée au début de cette étude : pourquoi exprimons-nous une quantité de liquide par un collocatif quantifieur, souvent figuratif ou métaphorique, au lieu de dire 'un peu de' ou 'un tout petit peu de'? Pourquoi « un petit café made in Guadeloupe, agrémenté d'une larme de lait de coco » paraît-il plus attrayant qu'un petit café avec un peu de lait ou une petite quantité de lait ? Pourquoi s'en sert-on lorsqu'on parle d'une quantité de termes abstraits ? Pourquoi quelqu'un invite à " une soirée avec un doigt de jazz, une pincée de pop, un zeste de rock» au lieu d'inviter à 'une soirée avec un peu de jazz, un peu de pop, un peu de rock' ?

Paradoxalement ou non, nous avons recours aux collocations lorsque nous voulons que nos pensées paraissent plus concrètes et en même temps soient plus précises. Et elles le paraissent plus quand elles sont exprimées par les collocatifs quantifieurs tels que une goutte de, une larme de, un doigt de, etc. que si elles étaient renfermées dans des locutions ordinaires comme 'une petite quantité de' ou 'une quantité minime de', bien que toutes les deux désignent une quantité faible. 
Cela résulte du fait qu'au sens usuel hérité d'Aristote la quantité est une notion abstraite. Les quantifieurs comme un kilo de, un mètre de, un litre de, etc. sont directement issus de l'abstraction. Les collocatifs qui font l'objet de cette étude sont occasionnellement quantifieurs. Ils correspondent, à l'origine, à des noms de choses véritables ou de phénomènes communément connus comme une larme, un doigt, une goutte, une trace, une gorgée, une pointe, etc. Ils bénéficient d'une autonomie référentielle, ce qui nous permet d'imaginer et d'exprimer, par référence à une réalité concrète, la notion abstraite qui est la quantité.

\section{En guise de conclusion}

Cette étude n'est pas exhaustive. Elle exige d'autres recherches encore plus approfondies et pertinentes mais nous semble avoir le mérite de bien montrer la combinatoire des collocatifs marquant une petite quantité (le plus souvent de liquide) en français actuel tout en précisant la valeur sémantique de ces collocatifs. Nous pouvons en tirer quelques conclusions.

Contrairement aux quantifieurs marquant une grande quantité indéterminée, tels une avalanche de (courriels), un flot de (voitures), un bataillon de (fourmis), une forêt de (bras levés), etc., ils n'acceptent pas de noms concrets comptables ; ils privilégient les noms concrets massifs non comptables mais quantifiables ( $c f$. vin, poudre, miel, etc.) et les noms abstraits par définition non comptables et non quantifiables ( $c f$. jalousie, tristesse, peur, jazz, rock, etc.).

Certains d'entre eux remplissent la fonction de collocatifs figuratifs ( $c f$. une larme de porto, un doigt de vodka), d'autres la fonction de collocatifs métaphoriques lorsqu'ils sont combinés avec des noms abstraits ( $c f$. une goutte d'amour, une gorgée de liberté, une trace d'espoir, une pointe de jalousie, etc.).

Ils acquièrent deux valeurs sémantiques fondamentales, soit une valeur quantitative (cf. une larme de porto, un nuage de lait), soit une valeur intensive (cf. un soupçon de jazz, une goutte de bonheur). Ils fonctionnent donc ou bien comme des quantifieurs marquant une petite quantité d'objets concrets massifs ou bien comme des intensifieurs (atténuateurs) marquant une intensité faible de la propriété.

\section{Bibliographie}

Asnès, Maria, "Quantification d'objets et d'événements : analyse contrastive des quantifieurs nominaux et des flexions verbales ", Langages, 2008, $n^{\circ} 169$, p. 82-91

Benninger, Céline, « Une meute de loups / une brassée de questions : collection, quantification et métaphore », Langue française, 2001, nº 129, p. 21-34 ou [en ligne] http://www.persee.fr/doc/ 1fr_0023-8368_2001_num_129_1_1015

Blanco, Xavier, « Les déterminants figés », Langages, 2002, nº 145, p. 61-80 ou [en ligne] http:// www.persee.fr/doc/lgge_0458-726x_2002_num_36_145_907 
Buvet, Pierre-André, "Les modifieurs des noms au regard du figement : le cas des groupes prépositionnels », 2012, [en ligne] https://hal.archives-ouvertes.fr/hal-00680254/document

Dessaux, Anne-Marie, « Déterminants nominaux et paraphrases prépositionnelles : Problèmes de description syntaxique et sémantique du lexique », Langue française, 1976, $\mathrm{n}^{\circ}$ 30, p. 44-62

Gaatone, David, « Esquisse d'un guide des perplexes : problèmes de définition et de classification des adverbes de degré en français », Langue Française, 2013, n 177, p. 37-50

Hausmann, Franz Josef, «Le dictionnaire de collocations » in Wörterbücher : ein internationales Handbuch zur Lexicographie. Dictionaries. Dictionnaires, éd. F.J. Hausmann, O. Reichmann, H. E. Wiegand, L. Zgusta, Berlin, De Gruyter, 1989, p. 1010-1019

Izert, Małgorzata, « Les quantifieurs nominaux d'origine météorologique ou hydrographique en français et en polonais », Neophilologica, 2018, vol. 30, p. 120-134

Leroy, Sarah, « D’un torrent de larmes à un Himalaya de bêtise. Sur certains déterminants nominaux métaphoriques en français », Travaux de linguistique, 2005, n 50, p. 97-112

Maingueneau, Dominique, La Syntaxe du français, Paris, Hachette, 1994

Mel'cuk, Igor et al., Dictionnaire explicatif et combinatoire du français contemporain. Recherches lexico-sémantiques. Vol. I, Montréal, Presses de l’Université de Montréal, 1984

$T L F i$ (s.d.). Trésor de la Langue Française informatisé [en ligne], http://atilf.atilf.fr/tlfi.htm http://www.google.fr, consulté entre le 6.05.2018 et le 31.05.2018

Małgorzata Izert - est maître de conférences habilitée à diriger les recherches au Département de linguistique à l'Institut d'Études Romanes, Université de Varsovie. Elle enseigne la linguistique générale, la sémantique, la lexicologie et la lexicographie, la grammaire descriptive et la grammaire historique de la langue française. Ses travaux de recherche portent sur le lexique, la phraséologie et les analyses linguistiques sur corpus. Depuis sa thèse de doctorat (2002) : Les Expressions Adj. comme $S N$ et intensification de la propriété elle analyse les différents moyens linguistiques servant à exprimer l'intensité, entre autres les adjectifs, les adverbes, les syntagmes binominaux : $\mathrm{N}_{1} \mathrm{~N}_{2}$, les collocations Adj./N/V à $S V_{\text {inf }}$ le suffixe -issime et les préfixes intensifieurs qui ont fait l'objet de sa thèse d'habilitation (2015) : La Construction préfixale de forte intensité en français contemporain, Łask, LEKSEM. Elle a participé au projet international : «Les comparaisons et l'intensification » dans le cadre du Programme Hubert Curien « Polonium 2015-2016». 